\title{
TECNOLOGÍA LÍTICA Y ESTRATEGIAS DE SUBSISTENCIA DURANTE LOS PERÍODOS ARCAICO Y FORMATIVO EN EL ALTIPLANO CENTRAL, BOLIVIA*
}

\author{
LITHIC TECHNOLOGY AND SUBSISTENCE STRATEGIES DURING THE \\ ARCHAIC AND FORMATIVE PERIODS IN THE CENTRAL ALTIPLANO, \\ BOLIVIA
}

\author{
José M. Capriles ${ }^{1}$, Sergio Calla Maldonado ${ }^{2}$ y Juan Albarracín-Jordán ${ }^{3}$
}

\begin{abstract}
Los cambios producidos en los sistemas de subsistencia de caza-recolección al cultivo de plantas y crianza de camélidos constituyen una de las transiciones más interesantes en la arqueología del altiplano andino. En este reporte se presentan resultados preliminares del análisis de patrones de asentamiento y materiales líticos recuperados en la región de Iroco, el margen noreste del Lago Uru-Uru, en el altiplano central de Bolivia. Los cambios producidos en los sistemas de asentamiento entre el período Arcaico (10.000-3.500 a.p.) y el período Formativo (3.500-1.600 a.p.) están relacionados con cambios en la organización económica y patrones de movilidad. Sin embargo, los cambios tecnológicos se encuentran enmarcados en una posible tradición de utilización de recursos y procesos de manufactura aplicados a los materiales líticos.
\end{abstract}

Palabras claves: Arcaico, Formativo, Wankarani, líticos, patrones de asentamiento.

Changes in subsistence systems from hunter-gathering to plant cultivation and animal husbandry are one of the least understood aspects in the archaeology of the Andean highlands. In this report we present preliminary results the analysis of settlement patterns and lithic materials recovered from the Iroco area, in the northeast margin of Lake Uru-Uru, in the central highlands of Bolivia. Changes in settlement systems between the Archaic Period (10,000-3,500 BP) and the Formative Period (3,500-1,600 BP) are related to changes in economic organization and mobility patterns. However, technological change is framed in a possible tradition of resource utilization and manufacturing processes applied to stone materials.

Key words: Archaic, Formative, Wankarani, lithics, settlement patterns.

La transición entre el período Arcaico (10.0003.500 a.p.) y el período Formativo (3.500-1.600 a.p.) en los Andes sur-centrales se caracteriza por la sedentarización de poblaciones humanas a partir del desarrollo e incorporación de tecnologías de subsistencia agrícola (Browman 1981; Hastorf 2008; Stanish 2003). En este reporte presentamos datos preliminares de patrones de asentamiento y tecnología lítica asociados con esta transición en el altiplano boliviano. Durante el período Formativo el altiplano fue escenario de varias manifestaciones culturales, entre el complejo arqueológico Wankarani, caracterizado por asentamientos en forma de montículos, conformados por superficies con estructuras circulares superpuestas y la presencia de esculturas líticas representando a efigies de camélidos (Ayala y Uribe 2003; Ayala et al. 2008; Bermann y Estévez Castillo 1995; Fox 2007; Janusek 2008; McAndrews 1998, 2001, 2005; Michel López 2008; Ponce Sanginés 1970).

Pese a que existe una insuficiencia de estudios, específicamente enfocados en entender a las poblaciones cazadoras-recolectoras que antecedieron el desarrollo del complejo Wankarani, varios investigadores han sugerido que durante el período Formativo, en el altiplano central boliviano, se consolidó un modo de vida agropastoril. Más aún, se desconoce la relación entre la organización económica y la tecnología de subsistencia asociada con grupos del período Arcaico y el complejo

* Un texto preliminar de este artículo fue presentado en el $53^{\circ}$ Congreso Internacional de Americanistas, Simposio "El período Arcaico en los Andes Sur Centrales: Tradiciones culturales e innovaciones tecnológicas", Ciudad de México, 19 al 24 de julio de 2009. Este manuscrito fue evaluado por investigadores externos y editado por Luis Flores Blanco y Mark S. Aldenderfer, en su calidad de editores invitados de la Revista.

1 Department of Anthropology, Washington University in St. Louis, One Brookings Drive, C.B. 1114, St. Louis, MO 63130. jcaprilesflores@wustl.edu

2 Carrera de Arqueología, Universidad Mayor de San Andrés, La Paz, Bolivia. sergioalejand@ gmail.com

3 Fundación Bartolomé de las Casas, La Paz, Bolivia. albarracinjordan@yahoo.com 
Wankarani y se advierte que se han interpolado procesos que derivan de investigaciones realizadas en las regiones vecinas del sur del Perú y norte de Chile cuyas evidencias materiales concretas todavía requieren verificación empírica (e.g., Aldenderfer 1989, 1998; Burger et al. 2000; Klink y Aldenderfer 2005; Núñez et al. 2005; Olivera 1997; Santoro 1989; Santoro y Núñez 1987). Por ejemplo, si bien se anticipa que los sistemas de producción de alimentos cambiaron notablemente, desconocemos si algunos patrones de la tecnología lítica persistieron, a pesar del cambio en la organización económica. En este sentido, el principal objetivo de este reporte consiste en ofrecer una primera interpretación sobre la transición arcaico-formativa en la región central del altiplano boliviano. Para ello se presentan los resultados del análisis de patrones de asentamiento, distribución de materias primas y tecnología lítica del área de Iroco, ubicada en el margen noreste del Lago Uru-Uru, en el Departamento de Oruro, en Bolivia.

\section{Materiales y Métodos}

El área de estudio se ubica entre las colinas y estribaciones montañosas, al oeste de la ciudad de Oruro, y las planicies lacustres de la orilla noreste del Lago Uru-Uru, a una altura que varía entre 3.690 y 4.050 msm (Albarracín-Jordán 2005; Capriles $2008,2011)$. Iroco se encuentra dentro del altiplano central y es parte de la puna seca, caracterizada por un clima semiárido de fuerte radiación solar y cobertura vegetal dispersa y xerofítica (pajonales y tolares), ocasionalmente interceptada por ríos y humedales de altura, conocidos como bofedales (Cuenca Sempertegui et al. 2005).

Una secuencia paleoclimática para el Río Desaguadero y los lagos Uru-Uru y Poopó ha sido propuesta por Rigsby y colaboradores (2005), utilizando una combinación de sedimentología, identificación de diatomeas y fechados radiocarbónicos. En correspondencia con reconstrucciones paleoclimáticas de zonas vecinas (e.g., Abbott et al. 1997; Núñez et al. 2005; Sylvestre et al. 1999; Thompson et al. 1998; Yacobaccio y Morales 2005), el altiplano central se caracteriza por periodos alternados de mayor y menor humedad identificados por la formación de paleolagos, paleorríos y erosión (Baucom y Rigsby 1999). En general, la secuencia altiplánica parece correlacionarse bien con fases húmedas a escala global como ser el Younger
Dryas, el evento ocurrido alrededor del 8.200 a.p. y el neoglaciar. Asimismo, el periodo entre 7.900 y 4.500 a.p. corresponde a condiciones de alta aridez (Rigsby et al. 2005). Con posterioridad a 4.500 a.p., el clima parece estabilizarse, pero sin dejar de incluir ocasionales inundaciones y sequías, tal como ocurre al presente (Fox 2007).

La prospección arqueológica realizada en Iroco consistió en la identificación de sitios empleando una estrategia de prospección sistemática de cobertura total y alta intensidad (Albarracín-Jordán 2005; Capriles 2011). El éxito alcanzado en previas investigaciones que emplearon estrategias de alta intensidad en otras regiones altiplánicas permitió predecir la identificación de numerosos sitios asociados con el Período Arcaico (Aldenderfer 1998; Cipolla 2005; Klink 2005). El equipo de prospección estuvo compuesto por cuatro a cinco personas, espaciadas a intervalos de 5 a 15 metros, procurando identificar toda evidencia de ocupación humana. Durante la prospección, los sitios arqueológicos fueron identificados a partir del hallazgo de concentraciones y dispersiones continuas a semicontinuas de materiales arqueológicos, incluyendo fragmentos de cerámica y de líticos tallados, así como la presencia de rasgos arquitectónicos, como ser restos de muros, terrazas y corrales.

En cada uno de los sitios identificados se procedió a registrar varios puntos en el centro y el perímetro, utilizando sistemas de posicionamiento global (GPS). Adicionalmente se tomaron fotografías digitales y se describieron las características particulares de cada sitio. Se recolectó material diagnóstico lítico y cerámico, procurando maximizar la inclusión de todos los tipos cerámicos y materias primas líticas presentes. La información fue posteriormente introducida en una base de datos y relacionada a un sistema de información geográfica. Los componentes (i.e., cronología y función) de los sitios asignados en campo fueron posteriormente corroborados en gabinete a partir de los resultados del análisis del material recolectado y complementado por los resultados de excavaciones arqueológicas en varios asentamientos (AlbarracínJordán 2005; Capriles 2008, 2011). La cronología fue determinada a partir de la identificación de atributos diagnósticos para componentes cronológicos concretos (Ayala y Uribe 2003; Ayala et al. 2008; Bermann y Estévez Castillo 1993; Fox 2007; Klink y Aldenderfer 2005; Lizárraga-Mehringer 2004; McAndrews 2005). 
El análisis de los artefactos líticos fue realizado a nivel macroscópico (Calla 2009). El análisis tecnológico se basó en la identificación de atributos individuales de cada pieza observada (Aldenderfer 1998; Andrefsky 2005, 2009; Odell 2003; Sackett 1989). La definición de materia prima incluyó una apreciación cualitativa de la distancia desde la zona del hallazgo hacia la probable fuente o cantera en base a mapas geológicos y nuestra experiencia en la región de estudio. Además de las dimensiones y materia prima, las categorías tecnomorfológicas utilizadas en el análisis incluyeron herramientas en lascas y láminas (raspadores, raederas, cuchillos, etc.), bifaces con y sin pedúnculo (e.g., puntas de proyectil, azadas, hachas de mano, etc.), otros artefactos (manos de moler, cuentas de collar, etc.) y productos de desecho de talla (debitage).

El desecho de talla fue incluido en el análisis debido a que proporciona elementos para entender la remoción secuencial de las piezas de descarte, las actividades de reducción de núcleos y la producción de herramientas, así como el lugar donde (las herramientas líticas) fueron confeccionadas y su posterior transporte, transformación (incluyendo retoque) o descarte (Andrefsky 2009). Asimismo, sobre la base de investigaciones previas, se elaboró una tipología de artefactos para el área de estudio como herramienta para ordenar la variabilidad identificada de una manera relacional y jerárquica (ver Aldenderfer 1998; Klink y Aldenderfer 2005).

\section{Resultados}

\section{Patrones de asentamiento}

La prospección cubrió una superficie continua de $38,35 \mathrm{~km}^{2}$, habiéndose identificado 185 sitios arqueológicos, los cuales fueron asignados a uno de los siguientes componentes: Arcaico, Formativo, Tiwanaku, Intermedio Tardío, Inca, Colonial, Republicano e Indeterminado. La densidad promedio de sitios arqueológicos en la zona de estudio fue de 4,82 sitios por $\mathrm{km}^{2}$, que podría considerarse como elevada. En total, 331 componentes fueron registrados (Capriles 2011).

Se identificaron 35 sitios con componentes correspondientes al período Arcaico o Precerámico (Figura 1). La densidad promedio registrada para este período fue de 0,91 sitios por $\mathrm{km}^{2}$. En conjunto, estos asentamientos cubren una superficie de 5,83 hectáreas, alcanzando una superficie promedio de
0,17 hectáreas $(\mathrm{SD}=0,34)$. Únicamente tres de estos sitios tienen más de media hectárea de superficie. La distribución de estos sitios está concentrada alrededor de una serie de colinas bajas, planicies y dunas ubicadas en las cercanías de la confluencia del Río Karakollu en el Lago Uru-Uru, en el sector norte del área de prospección.

Un total de 45 sitios fueron registrados con ocupaciones correspondientes al período Formativo (Figura 2). La superficie acumulada de estos sitios alcanza las 14,51 hectáreas. El tamaño promedio de los sitios es de 0,32 hectáreas $(\mathrm{SD}=0,48)$ y, aproximadamente, diez de estos sitios superan la media hectárea de extensión. La densidad de asentamientos registrada asciende a 1,17 sitios por $\mathrm{km}^{2}$, ligeramente superior a la del período precedente. $\mathrm{La}$ distribución de asentamientos es más dispersa que en el período Arcaico, incluyendo agrupamientos relativamente más separados pero manteniendo una relativa asociación con la orilla del Lago Uru-Uru. Los sitios de mayor extensión presentan claras evidencias de ocupación doméstica, mientras que los restantes ( 35 sitios) posiblemente conformaron una serie de residencias o campamentos base. Es interesante observar que únicamente seis sitios incluyen componentes arcaicos y formativos. Estos sitios se encuentran entre los más grandes y, en conjunto, ocupan 3,85 hectáreas.

Excavaciones arqueológicas en el sitio $\mathrm{KCH} 20$ permitieron la identificación de un fogón y materiales arqueológicos asociados con una ocupación datada al período Arcaico Temprano (Capriles 2011). De hecho, dos fechados radiocarbónicos AMS proporcionaron una edad entre 9.289 y 8.729 años cal. a.p. (8.273 \pm 82 a.p., AA91568 colágeno de hueso $\mathrm{d}^{13} \mathrm{C}=-19,7 \%$ y $8.105 \pm 92$ a.p., AA91569, colágeno de hueso $\mathrm{d}^{13} \mathrm{C}-20 \%$ o). Este rasgo contenía 446 fragmentos óseos (incluyendo especímenes de guanacos, cuyes, ciervos, gallinetas y patos) y 155 fragmentos líticos que incluían al menos tres puntas de proyectil (ver Figura 4a, h, m). Excavaciones arqueológicas en sitios formativos (cuya asignación cronológica fue confirmada mediante numerosos fechados radiocarbónicos) permitieron la recuperación de centenares de fragmentos cerámicos, líticos y óseos (donde predominaban los restos de camélidos, peces y aves acuáticas) (Capriles 2011). La muestra más grande corresponde al sitio $\mathrm{KCH} 21$, fechado aproximadamente entre 2.290 y 1.730 cal. a.p., donde se identificaron varios de tipos de puntas 


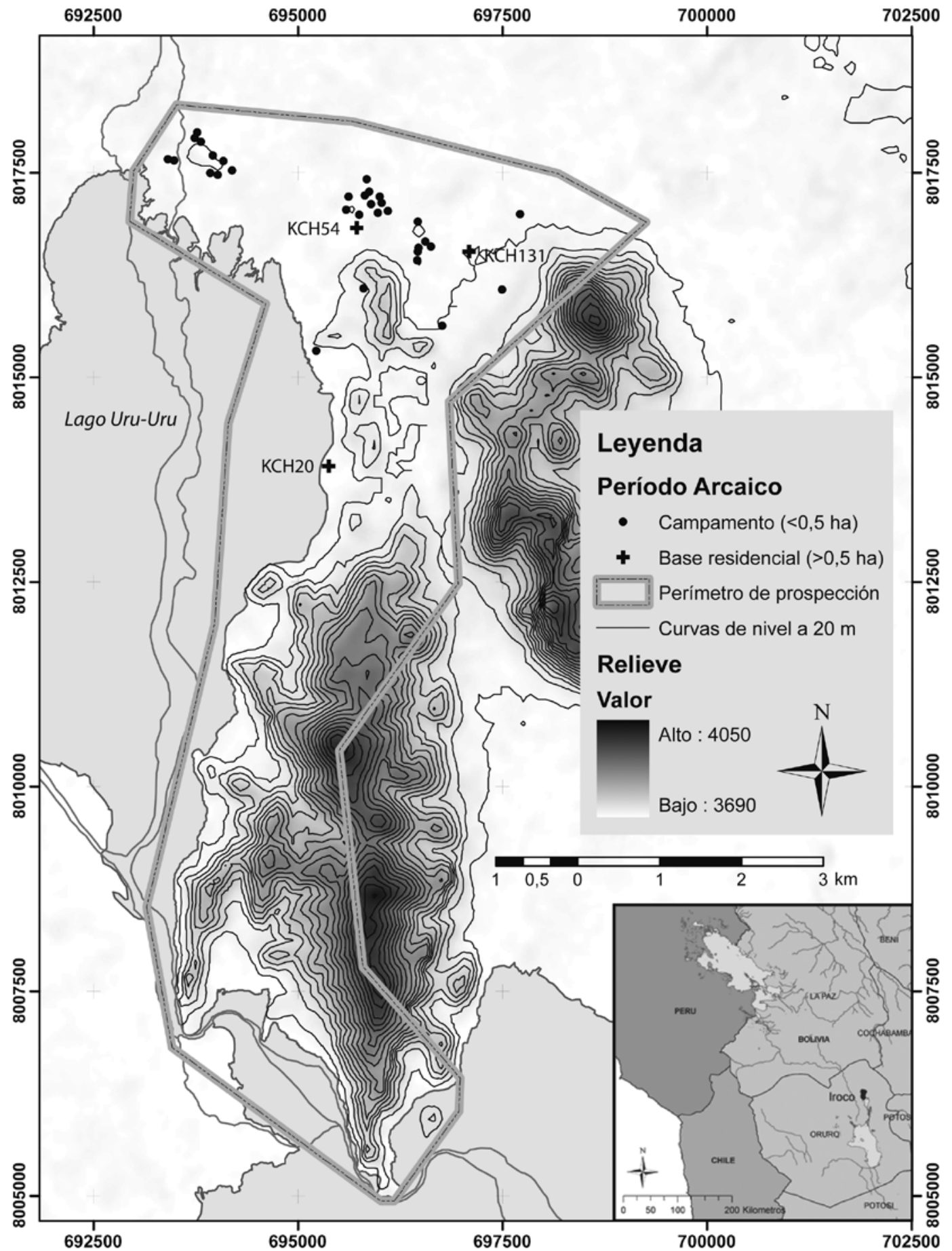

Figura 1. Patrón de asentamiento del período Arcaico en Iroco, Bolivia.

Settlement pattern of the Archaic Period in Iroco, Bolivia. 


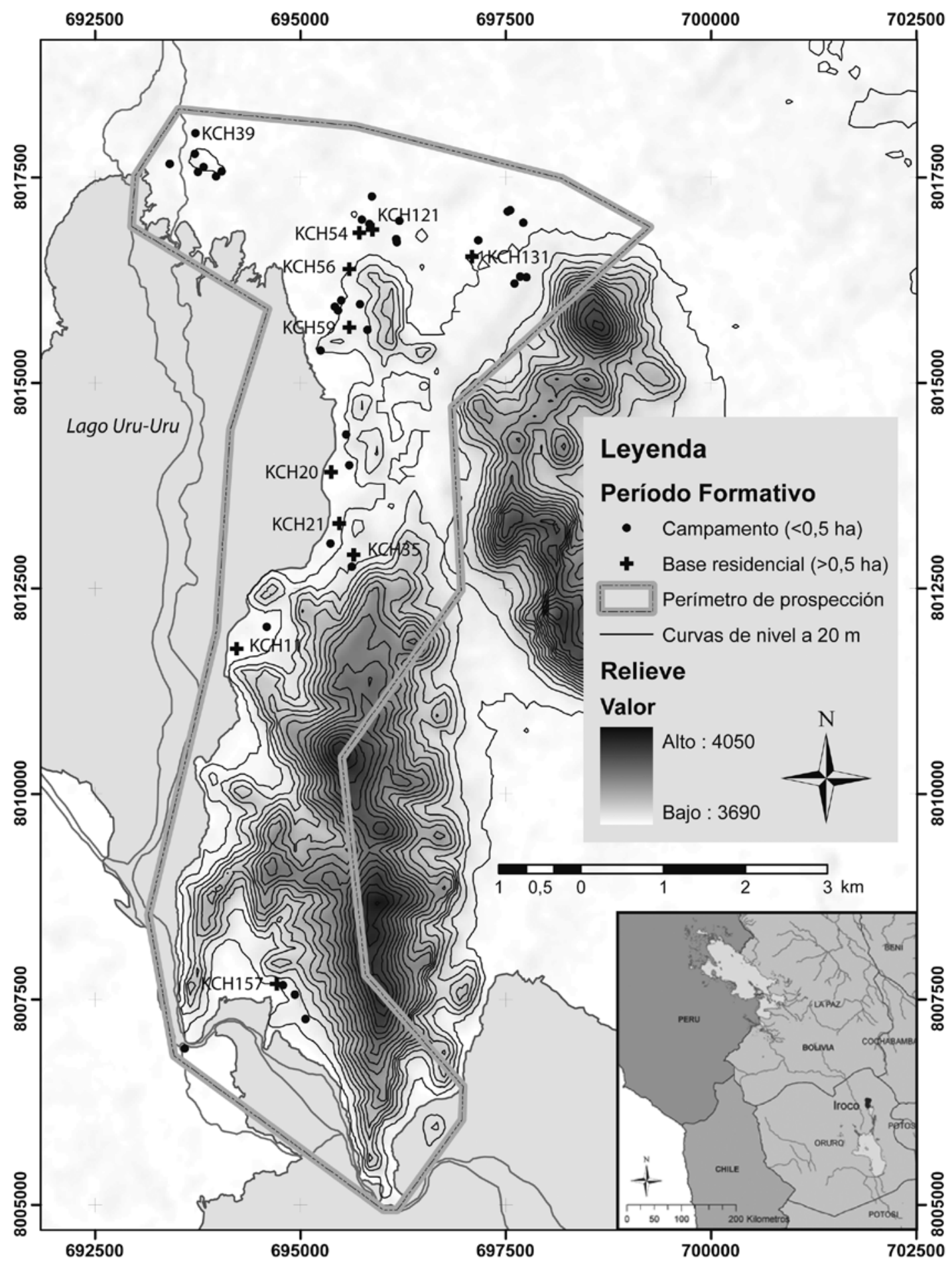

Figura 2. Patrón de asentamientos del período Formativo en Iroco, Bolivia. Settlement pattern of the Formative Period in Iroco, Bolivia. 
de proyectil en contextos culturales típicamente domésticos (ver Figura 5b-g, 5n-s, 5x).

\section{Materias primas}

La muestra analizada consistió de 1.058 especímenes líticos procedentes de colecciones de superficie, de los cuales 500 pertenecen a 29 sitios arcaicos, 369 a 29 sitios formativos y 189 a cinco sitios con la presencia de ambos componentes. El conjunto lítico incluye la representación de al menos 14 distintos tipos de materias primas (Tabla 1). Las materias primas más frecuentes son de grano fino, origen no local, y corresponden a basalto negro y sílex oscuro (sílices principalmente negros y grises). Otras materias primas, no locales, incluyen basalto gris y obsidiana que ocurren muy esporádicamente. Las materias primas de origen local incluyen sílex claro (compuesto por sílices de color blanco, amarillo y café), cuarcita, cuarzo lechoso, calcedonia, pizarra, arenisca cuarcítica y cuarzo cristalino. Sin embargo, durante el Arcaico estas materias primas no fueron empleadas para la confección de herramientas con tanta intensidad como las materias primas de origen no local. Esto contrasta con el período Formativo, cuando los recursos disponibles localmente fueron utilizados con mayor frecuencia, algunos de éstos, claramente de menor calidad. Aún así, persistió el uso de materias primas no locales, incluyendo algunas de muy buena calidad (e.g., obsidiana, jaspe y sodalita).

\section{Tecnología lítica}

Las categorías tecnofuncionales están representadas de manera similar en los sitios de ambos períodos con excepción del debitage (ver más adelante) (Tabla 2). Otras excepciones incluyen la aparición de azadas durante el período Formativo junto con una mayor frecuencia de raspadores bifaciales y núcleos. En contraste, los sitios del período Arcaico presentan una mayor proporción de raspadores unifaciales (Figura 3). Asimismo, mientras algunos raspadores unifaciales fueron elaborados en láminas, la mayoría (al igual que todos los raspadores bifaciales y todos los cuchillos) se manufacturaron en lascas. Por otro lado, las azadas, únicamente presentes en sitios formativos, presentan forma ojival o elíptica y varían entre 5 y $18 \mathrm{~cm}$ de altura.

Basados en un análisis morfológico construimos una tipología de puntas de proyectil que además se encuentra apoyada en materiales derivados no solamente de superficie sino también de excavaciones arqueológicas (Figuras 4-5, Tabla 3). Los tipos tempranos de puntas de proyectil con pedúnculo más frecuentes tienen morfología triangular (Figura 4b-e) y romboidal (Figura 4f-i) con los lados de la hoja rectos mientras que los tipos más comunes de puntas de proyectil sin pedúnculo tienen forma ojival (Figura 4k-1) y triangular (Figura 4n-o). Tres puntas de proyectil (Figura $4 \mathrm{a}, \mathrm{h}, \mathrm{m}$ ) correspondientes al fogón del sitio $\mathrm{KCH} 20$ y datadas entre el 9.289 y 8.729 a.p. permiten confirmar su asignación al

Tabla 1. Materias primas de líticos identificados en los sitios del área de estudio. Lithic raw materials identified in the sites of the study area.

\begin{tabular}{llrrrrrr}
\hline Materia Prima & Origen & Arcaico & \multicolumn{1}{c}{$\%$} & Ambos & $\%$ & Formativo & $\%$ \\
\hline Basalto negro & No local & 311 & 62,2 & 119 & 63 & 177 & 48 \\
Sílex oscuro & No local & 132 & 26,4 & 42 & 22,2 & 83 & 22,5 \\
Sílex claro & Local & 28 & 5,6 & 3 & 1,6 & 23 & 6,2 \\
Cuarcita & Local & 9 & 1,8 & 9 & 4,8 & 23 & 6,2 \\
Cuarzo lechoso & Local & 6 & 1,2 & 0 & 0 & 1 & 0,3 \\
Calcedonia & Local & 5 & 1 & 0 & 0 & 5 & 1,4 \\
Basalto gris & No local & 3 & 0,6 & 9 & 4,8 & 17 & 4,6 \\
Obsidiana & No local & 2 & 0,4 & 3 & 1,6 & 19 & 5,1 \\
Pizarra & Local & 2 & 0,4 & 1 & 0,5 & 6 & 1,6 \\
Arenisca cuarcítica & Local & 1 & 0,2 & 1 & 0,5 & 1 & 0,3 \\
Cuarzo cristalino & Local & 1 & 0,2 & 0 & 0 & 1 & 0,3 \\
Jaspe & No local & 0 & 0 & 1 & 0,5 & 9 & 2,4 \\
Arenisca & Local & 0 & 0 & 1 & 0,5 & 1 & 0,3 \\
Sodalita & No local & 0 & 0 & 0 & 0 & 3 & 0,8 \\
Total & & 500 & 100 & 189 & 100 & 369 & 100 \\
\hline
\end{tabular}


Tabla 2. Categorías tecnofuncionales identificadas en el área de estudio. Techno-functional categories identified in the study area.

\begin{tabular}{lcccccc}
\hline Tipo de artefacto & Arcaico & $\%$ & Ambos & $\%$ & Formativo & $\%$ \\
\hline Punta de proyectil con pedúnculo & 23 & 4,6 & 13 & 6,9 & 22 & 6,0 \\
Punta de proyectil sin pedúnculo & 29 & 5,8 & 19 & 10,1 & 26 & 7,0 \\
Raspador unifacial en lasca & 83 & 16,6 & 39 & 20,6 & 77 & 20,9 \\
Raspador unifacial en lámina & 8 & 1,6 & 3 & 1,6 & 3 & 0,8 \\
Raspador bifacial & 2 & 0,4 & 2 & 1,1 & 14 & 3,8 \\
Cuchillo unifacial & 6 & 1,2 & 1 & 0,5 & 3 & 0,8 \\
Cuchillo bifacial & 0 & 0 & 0 & 0 & 3 & 0,8 \\
Buril & 5 & 1 & 0 & 0 & 5 & 1,4 \\
Azada & 0 & 0 & 11 & 5,8 & 27 & 7,3 \\
Núcleo & 7 & 1,4 & 2 & 1,1 & 13 & 3,5 \\
Pebble & 2 & 0,4 & 0 & 0 & 0 & 0 \\
Mano de moler & 1 & 0,2 & 0 & 0 & 0 & 0 \\
Cuenta de collar & 0 & 0 & 0 & 0 & 1 & 0,3 \\
Debitage & 334 & 66,8 & 99 & 52,4 & 175 & 47,4 \\
Total & 500 & 100 & 189 & 100 & 369 & 100 \\
\hline
\end{tabular}

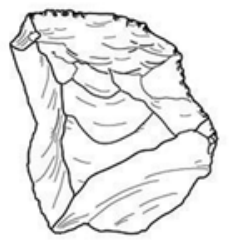

a
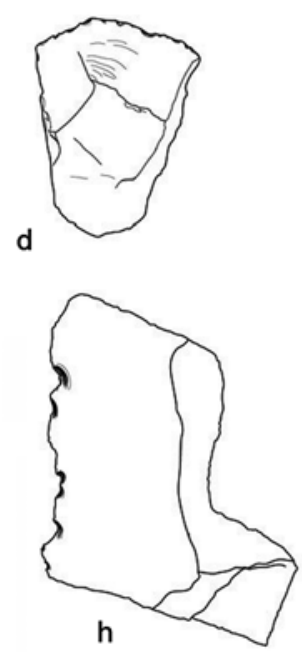

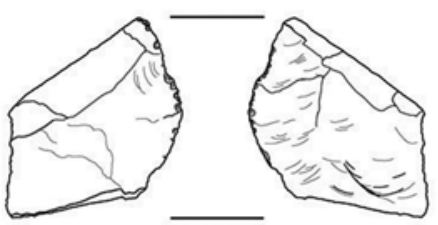

b

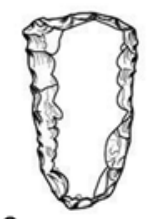

e
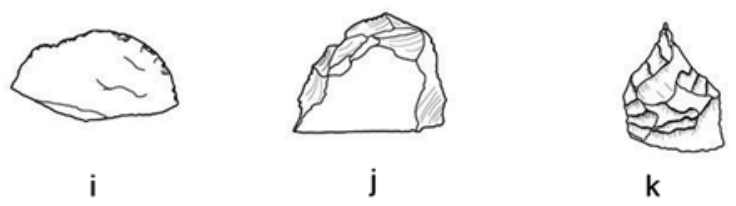

C
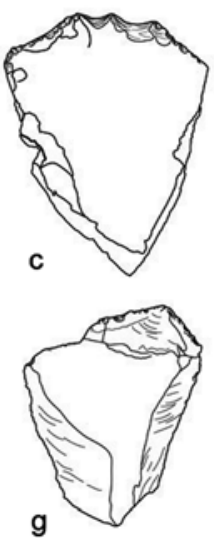

k

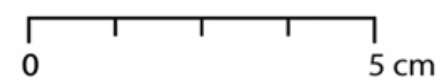

Figura 3. Tipos de raspadores: (a-b) raspadores laterales, (c-g) raspadores terminales, (h) denticulado, (i) semilunar, (j) de uña, (k) buril simple. Todos los ejemplos proceden de sitios pertenecientes al período Arcaico.

Scraper types: ( $a$-b) lateral scrapers, (c-g) terminal scrapers, $(h)$ denticulate, $(i)$ semi-lunar, $(j)$ thumbnail, $(k)$ simple burin. All the examples are from Archaic Period sites. 
Tabla 3. Descripción de puntas de proyectil características del área de estudio (ver Figuras 4 y 5) Description of projectile points characteristics of the study area (see Figures 4 and 5)

\begin{tabular}{|c|c|c|c|}
\hline Figura & Sitio & Descripción morfológica & Período \\
\hline $4 \mathrm{a}$ & $\begin{array}{l}\mathrm{KCH} 20, \\
\text { Excavación }\end{array}$ & $\begin{array}{l}\text { Romboidal, medianamente espesa, de tamaño mediano, hoja de } \\
\text { lados rectos con hombros en los extremos, pedúnculo trapezoidal } \\
\text { con base recta }\end{array}$ & Arcaico Temprano \\
\hline $4 \mathrm{~b}-\mathrm{e}$ & $\mathrm{KCH} 20$, Superficie & $\begin{array}{l}\text { Triangular, medianamente espesa, de tamaño mediano y pequeño, } \\
\text { hoja de lados rectos, pedúnculo trapezoidal con base semiconvexa } \\
\text { o convexa }\end{array}$ & $\begin{array}{l}\text { Arcaico Temprano- } \\
\text { Arcaico Medio }\end{array}$ \\
\hline $4 \mathrm{f}$ & $\mathrm{KCH} 20$, Superficie & $\begin{array}{l}\text { Romboidal, medianamente espesa, de tamaño pequeño, hoja de lados } \\
\text { semiconvexos, pedúnculo trapezoidal con base convexa }\end{array}$ & $\begin{array}{l}\text { Arcaico Temprano- } \\
\text { Arcaico Medio }\end{array}$ \\
\hline $4 \mathrm{~g}$ & KCH20, Superficie & $\begin{array}{l}\text { Triangular, medianamente espesa, de tamaño grande, hoja de lados } \\
\text { rectos, pedúnculo trapezoidal con base recta }\end{array}$ & $\begin{array}{l}\text { Arcaico Temprano- } \\
\text { Arcaico Medio }\end{array}$ \\
\hline $4 \mathrm{~h}$ & $\begin{array}{l}\mathrm{KCH} 20 \\
\text { Excavación }\end{array}$ & $\begin{array}{l}\text { Triangular, medianamente espesa, de tamaño mediano, hoja de lados } \\
\text { rectos, pedúnculo trapezoidal con base recta }\end{array}$ & Arcaico Temprano \\
\hline $4 \mathrm{i}$ & $\mathrm{KCH} 20$, Superficie & $\begin{array}{l}\text { Triangular, medianamente espesa, de tamaño mediano, hoja de lados } \\
\text { rectos, pedúnculo trapezoidal con base convexa }\end{array}$ & $\begin{array}{l}\text { Arcaico Temprano- } \\
\text { Arcaico Medio }\end{array}$ \\
\hline $4 \mathrm{j}$ & $\mathrm{KCH} 20$, Superficie & $\begin{array}{l}\text { Triangular apedunculada, medianamente espesa, presenta hoja con } \\
\text { lados rectos y truncamiento en la base }\end{array}$ & Arcaico Medio \\
\hline $4 \mathrm{k}-1$ & $\mathrm{KCH} 20$, Superficie & $\begin{array}{l}\text { Ojival apedunculada, medianamente espesa y gruesa, de tamaño } \\
\text { grande, presenta hoja con lados semiconvexos retoque denticulado }\end{array}$ & Arcaico Medio \\
\hline $4 \mathrm{~m}$ & $\begin{array}{l}\mathrm{KCH} 20 \\
\text { Excavación }\end{array}$ & $\begin{array}{l}\text { Ojival apedunculada, medianamente espesa, de tamaño mediano, } \\
\text { presenta hoja con lados semiconvexos }\end{array}$ & $\begin{array}{l}\text { Arcaico Temprano- } \\
\text { Arcaico Medio }\end{array}$ \\
\hline $4 n-0$ & $\mathrm{KCH} 20$, Superficie & $\begin{array}{l}\text { Triangular alargada, apediculada, delgada, de tamaño mediano y } \\
\text { pequeño, hoja con lados rectos, base recta }\end{array}$ & $\begin{array}{l}\text { Arcaico Temprano- } \\
\text { Arcaico Medio }\end{array}$ \\
\hline $5 \mathrm{a}$ & KCH54, Superficie & Ojival, apedunculada, medianamente espesa, de tamaño mediano & $\begin{array}{l}\text { Arcaico Tardío- } \\
\text { Terminal/Formativo }\end{array}$ \\
\hline $5 \mathrm{~b}$ & $\begin{array}{l}\mathrm{KCH} 21, \\
\text { Excavación }\end{array}$ & $\begin{array}{l}\text { Elipsoide, apedunculada, medianamente espesa, de tamaño mediano } \\
\text { y pequeño }\end{array}$ & $\begin{array}{l}\text { Arcaico Tardío- } \\
\text { Terminal/Formativo }\end{array}$ \\
\hline $5 \mathrm{c}$ & $\begin{array}{l}\mathrm{KCH} 21, \\
\text { Excavación }\end{array}$ & $\begin{array}{l}\text { Ojival apedunculada, medianamente espesa y delgada, de tamaño } \\
\text { mediano y pequeño }\end{array}$ & $\begin{array}{l}\text { Arcaico Tardío- } \\
\text { Terminal/Formativo }\end{array}$ \\
\hline $5 \mathrm{~d}-\mathrm{e}$ & $\begin{array}{l}\mathrm{KCH} 21, \\
\text { Excavación }\end{array}$ & $\begin{array}{l}\text { Ojival apedunculada, medianamente espesa, de tamaño mediano } \\
\text { y pequeño }\end{array}$ & Formativo \\
\hline $5 f-g$ & $\begin{array}{l}\mathrm{KCH} 21, \\
\text { Excavación }\end{array}$ & $\begin{array}{l}\text { Triangular, gruesas o medianamente espesa, hoja de lados rectos o } \\
\text { semiconvexos, con muesca en la base }\end{array}$ & $\begin{array}{l}\text { Arcaico Tardío- } \\
\text { Terminal/Formativo }\end{array}$ \\
\hline $5 \mathrm{~h}-\mathrm{i}$ & $\begin{array}{l}\mathrm{KCH} 20, \mathrm{KCH} 39, \\
\text { Superficie }\end{array}$ & $\begin{array}{l}\text { Triangular alargada y apedunculada, medianamente espesa y delgada, } \\
\text { hoja de lados rectos o semiconvexos, base semicóncava }\end{array}$ & Formativo \\
\hline $5 \mathrm{j}-1$ & KCH20, Superficie & $\begin{array}{l}\text { Triangular alargada, medianamente espesa, hoja de lados rectos, pedún- } \\
\text { culo trapezoidal de tamaño variable y base semiconvexa y recta }\end{array}$ & Formativo \\
\hline $5 \mathrm{~m}$ & KCH39, Superficie & $\begin{array}{l}\text { Triangular, medianamente espesa, hoja de lados semiconvexos, } \\
\text { pedúnculo corto y base recta }\end{array}$ & Formativo \\
\hline $5 \mathrm{n}-\mathrm{p}$ & $\begin{array}{l}\mathrm{KCH} 21, \\
\text { Excavación }\end{array}$ & $\begin{array}{l}\text { Triangular, medianamente espesa, de tamaño mediano o pequeño, } \\
\text { hoja de lados semiconvexos, pedúnculo trapezoidal mediano con } \\
\text { base recta }\end{array}$ & Formativo \\
\hline $5 q-r$ & $\begin{array}{l}\mathrm{KCH} 21, \\
\text { Excavación }\end{array}$ & $\begin{array}{l}\text { Triangular, medianamente espesa, hoja de lados rectos y apéndices en } \\
\text { los extremos, pedúnculo grande o mediano, con base semiconvexa }\end{array}$ & Formativo \\
\hline $5 \mathrm{~s}$ & $\begin{array}{l}\mathrm{KCH} 21, \\
\text { Excavación }\end{array}$ & $\begin{array}{l}\text { Triangular, de lados seconvexos, medianamente espesa, tamaño } \\
\text { mediano, pedúnculo triangular }\end{array}$ & Formativo \\
\hline $5 \mathrm{t}-\mathrm{w}$ & $\begin{array}{l}\mathrm{KCH} 21, \mathrm{KCH} 54, \\
\text { Superficie }\end{array}$ & $\begin{array}{l}\text { Triangular, medianamente espesa, de tamaño pequeño, hoja semiconvexa } \\
\text { o recta, pedúnculo variable según subtipo, base semiconvexa }\end{array}$ & Formativo \\
\hline $5 \mathrm{x}$ & $\begin{array}{l}\mathrm{KCH} 21, \\
\text { Excavación }\end{array}$ & Elipsoide, medianamente espesas, de tamaño mediano y pequeño & Formativo \\
\hline
\end{tabular}




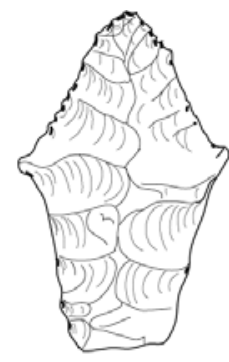

a

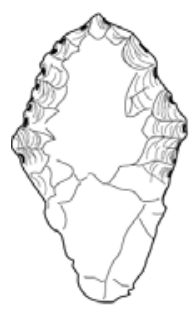

f

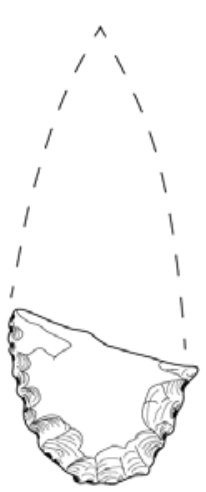

k

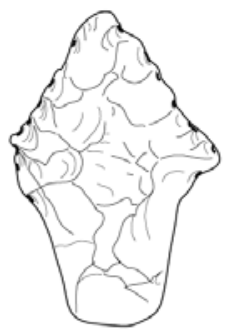

b

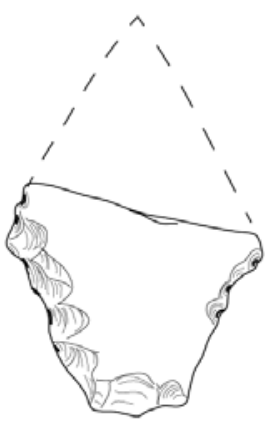

g

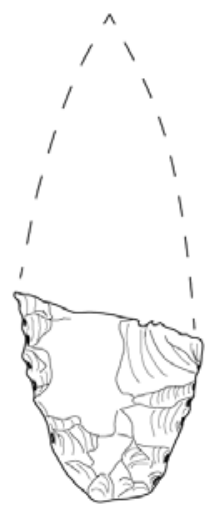

।

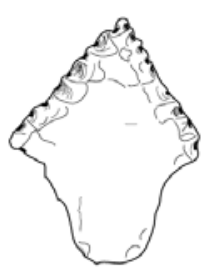

C

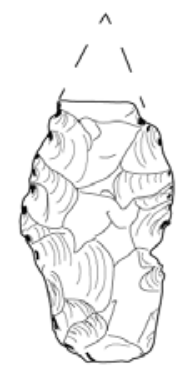

$\mathrm{h}$

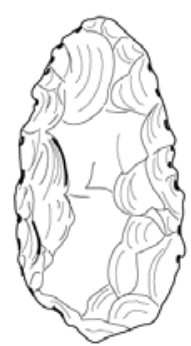

$\mathrm{m}$

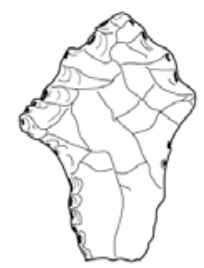

d

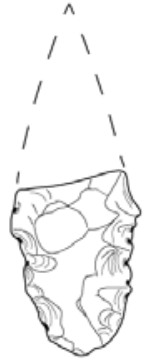

i
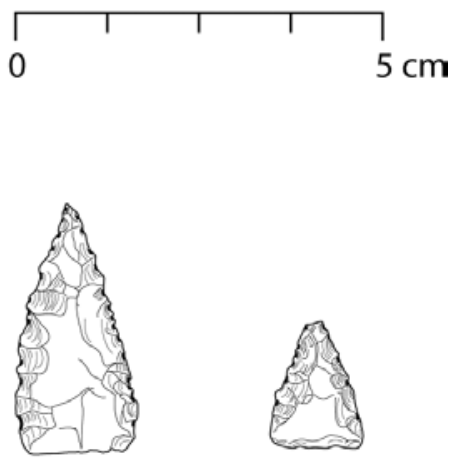

$\mathrm{n}$

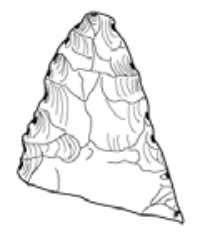

j

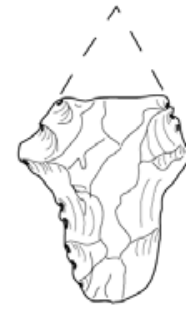

e $5 \mathrm{~cm}$

Figura 4. Puntas de proyectil de los períodos Arcaico Temprano y Medio (ver Tabla 3).

Early and Middle Archaic Period projectile points (see Table 3).

período Arcaico Temprano. Estos tipos son también comunes para el Arcaico Temprano y Arcaico Medio en el área Centro Sur Andina (Klink y Aldenderfer 2005; Lizárraga-Mehringer 2004; Santoro y Núñez 1987). Puntas de proyectiles similares también han sido encontradas en el sitio Viscachani (LizárragaMehringer 2004; Vela 1964).

Por otro lado, los sitios asociados con el período Formativo (y algunos del período Arcaico Tardío y
Terminal) incluyen una mayor variedad de puntas de proyectil, principalmente derivadas de formas elipsoides (Figura 5b, x), ojivales (Figura 5c-e), triangulares con pedúnculo con un gran espectro de subtipos (Figura 5j-w) y triangulares con muesca o base semiconvexa (Figura 5f-g). Comparativamente estas últimas son similares a las puntas de proyectil de los sitios de Hakenasa y Quelcatani (Klink y Aldenderfer 2005: Fig 3.6) datadas desde 


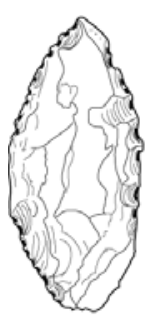

a

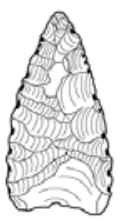

$\mathrm{h}$

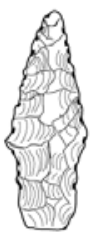

o
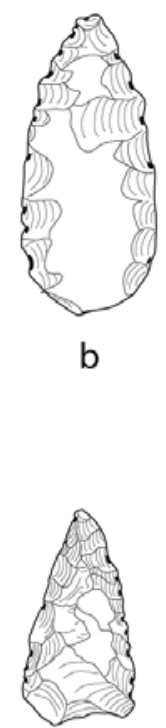

b

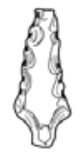

p

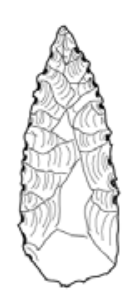

C

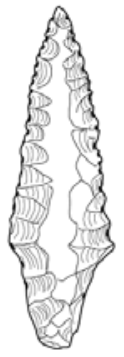

j

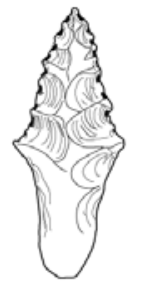

q

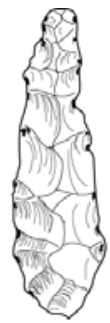

d
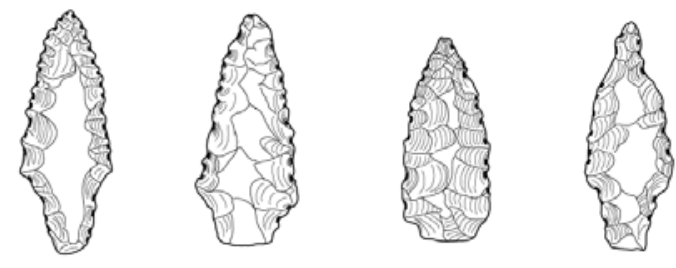

m
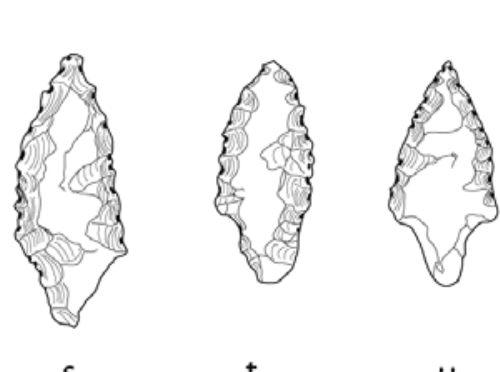

S
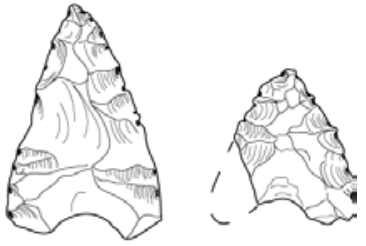

g

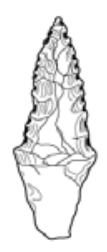

r

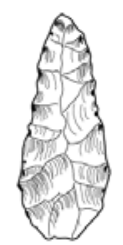

e n

u

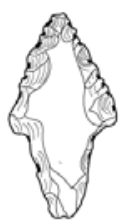

v

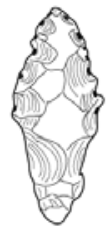

W

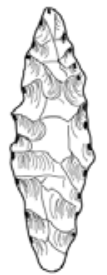

$\mathrm{X}$

Figura 5. Puntas de proyectil de los períodos Arcaico Tardío-Terminal y Formativo (ver Tabla 3).

Late-Terminal Archaic and Formative Period projectile points (see Table 3).

aproximadamente 4.400 al 1.500 a.p. para el Arcaico Terminal y el Formativo. También son comparables a las puntas de proyectil reportadas para el sitio Wankarani por Ponce Sanginés (1970:29, Fig.16) y Walter (1966:79, Fig. 10).

Gran parte de la muestra total está conformada por debitage (i.e., desecho de talla), que es mucho más frecuente en los sitios del período Arcaico que en los sitios del período Formativo. Algunas piezas de desecho tenían evidencia de retoque. De la muestra de debitage, las piezas marginales $(22,7 \%)$, lascas completas $(22,4 \%)$ y lascas proximales $(20,1 \%)$ son las más frecuentes. En menor proporción se tienen lascas mediales $(7,2 \%)$, astillas $(7,1 \%)$ y lascas longitudinales $(5,4 \%)$. Las láminas (completas y fragmentadas) representan el 11,3\% de la muestra, dos tercios de la cual provienen de sitios arcaicos. 


\section{Discusión}

Pese a la baja resolución temporal, los resultados obtenidos, en cuanto se refiere a frecuencia y densidad de asentamientos, son similares a los reportados por Cipolla (2005) y Klink (2005) en las orillas del lago Titicaca y difieren de otras prospecciones realizadas en regiones cercanas, donde los sitios precerámicos son infrecuentes (e.g., Albarracín-Jordán 1996; Stanish et al. 2002). Asimismo, nuestros resultados difieren notablemente de aquellos reportados por McAndrews (1998, 2001 , 2005) para la vecina región de La Joya, donde solamente 18 sitios formativos fueron registrados y ninguno del período Arcaico. Es probable que la baja frecuencia de asentamientos registrados por McAndrews ( 0,04 sitios por $\left.\mathrm{km}^{2}\right)$ se deba, en parte, a la baja intensidad de su prospección.

La mayor parte de los sitios pertenecientes al período Arcaico fueron identificados en el sector norte del área de estudio, donde su densidad varía entre 5 a 15 sitios por $\mathrm{km}^{2}$. Este sector se caracteriza por la presencia de numerosas dunas así como algunas colinas que interrumpen la planicie y que probablemente sirvieron como rompevientos y refugios. La ocasional presencia de afloramientos de sílex, cuarcita y cuarzo en estas colinas parece haber jugado un importante rol en el emplazamiento de los sitios. Sin embargo, la heterogeneidad de las dimensiones y conjuntos líticos asociados con cada sitio sugiere que podrían estar compuestos por múltiples ocupaciones temporales, campamentos logísticos y pequeños talleres. Asimismo, la cercanía de varios campamentos a la orilla lacustre sugiere que los recursos acuáticos tuvieron una gran importancia.

El sistema de asentamiento del período Formativo marca un importante contraste con el período Arcaico. Si bien las planicies y laderas bajas continúan siendo áreas preferenciales, el distanciamiento entre grupos de asentamientos parece incrementarse. Asimismo, se observa la colonización de nuevas áreas, tanto hacia el interior (este) de las quebradas y colinas como hacia la planicie aluvial (sur), posiblemente en asociación con recursos específicos (e.g., fuentes de agua o mejores suelos para la agricultura). Pese a que el incremento de sitios entre los períodos Arcaico y Formativo fue de 10 sitios, es decir, un incremento del $29 \%$, el incremento en superficie ocupada fue de 8,68 hectáreas, es decir 149\%. Gran parte de estos sitios también incluyen densidades altas de artefactos, arquitectura en superficie (cimientos de estructuras semicirculares) y la conformación de montículos. Interesantemente, los tres sitios arcaicos que superan las 0,5 hectáreas corresponden a sitios que también tienen ocupaciones formativas. Posiblemente, estos sitios conformaron campamentos base y fueron el sustrato material que permitió la estructuración del sistema de asentamientos durante el Formativo.

Es importante tomar en cuenta que pese a la baja resolución temporal de la asignación cronológica, existieron períodos en los que las condiciones ambientales pudieron incentivar o inhibir la ocupación humana. Utilizando la secuencia paleoclimática presentada por Rigsby y colaboradores (2005), podemos proponer que las condiciones de aridez identificadas entre 7.900 y 4.500 a.p. pudieron limitar el establecimiento de poblaciones humanas sustanciales (ver Moreno et al. 2009; Núñez et al. 2005; Yacobaccio y Morales 2005). Consecuentemente, las principales ocupaciones del Arcaico corresponderían a lapsos anteriores o posteriores a esta fase. Es con la mejora de las condiciones medioambientales que se produce la transición hacia el período Formativo. Interesantemente, el único asentamiento correspondiente al período Arcaico datado por radiocarbono en el área de estudio (KCH20) fue ocupado con anterioridad a este prolongado evento de aridez. El sitio $\mathrm{KCH} 20$ ocupado hace aproximadamente 9.200 años es además, uno de los asentamientos más antiguos investigados arqueológicamente en territorio boliviano.

A partir del análisis de los conjuntos líticos se puede inferir que la talla estuvo principalmente orientada hacia el uso de dos materias primas de origen no local, el basalto negro y el sílex oscuro. La fuente de basalto más conocida en Oruro es Querimita, que se ubica en la orilla suroeste del Lago Poopó (Ponce Sanginés y Mogrovejo Terrazas 1970). Querimita fue una de las canteras más utilizadas durante los períodos Formativo y Tiwanaku en la cuenca del lago Titicaca (Bermann y Estévez Castillo 1993; Giesso 2003). Sin embargo, existen otras canteras que también fueron importantes en el contexto regional (Giesso et al. 2003). Por ejemplo, reconocimientos recientes sugieren que los cerros Santa Bárbara y Casca Collu en la orilla sur del lago Poopó fueron importantes fuentes para la extracción de basalto negro y gris (Giesso et al. 2003; Michel López 2008). Por otro lado, existen algunas fuentes de sílex oscuro hacia el este de Iroco, en 
afloramientos rocosos que preceden la formación de los valles interandinos de Cochabamba.

Estudios recientes han provisto una cronología tipológica para los Andes sur centrales (Klink y Aldenderfer 2005). Varios tipos de puntas de proyectiles romboidales, ojivales y triangulares, identificadas por investigadores en otras regiones, presentan similitudes con las puntas de proyectil encontradas en Iroco. Tentativamente, estos hallazgos sitúan a nuestros artefactos en el período Arcaico Temprano, Medio y Tardío-Terminal. Puntas de proyectil comparables a las encontradas en los sitios del área de estudio han sido encontradas en sitios como Viscachani (Ibarra Grasso 1965; LizárragaMehringer 2004; Patterson y Heizer 1965; Vela 1964), Asana, Quelcatani (Aldenderfer 1998), Patapatane, Hakenasa (Santoro 1989; Santoro y Nuñez 1987), Ascotán 6 y Tambillo (Núñez et al. 2005) y algunos hallazgos encontrados en Huancané-Putina (Cipolla 2005). Las puntas de proyectil asociadas con el período Formativo muestran una importante variabilidad y son tecnológica y estilísticamente similares a ejemplares encontrados en otros sitios asociados con el complejo arqueológico Wankarani (Ayala et al. 2008; Ponce Sanginés 1970; Walter 1966). No obstante, a lo largo de la prehistoria de los Andes sur centrales es claro que existen importantes diferencias regionales (De Souza 2004; Klink y Aldenderfer 2005; Lizárraga-Mehringer 2004; Núñez et al. 2005).

De acuerdo a las características de los conjuntos líticos en la región, existe cierta estandarización y variabilidad tecnológica, conforme a cada período. Sin embargo, varios grupos artefactuales del Arcaico y el Formativo permanecieron sin muchos cambios a través del tiempo, especialmente ciertas herramientas (de manufactura expeditiva) como los raspadores y cuchillos. Asimismo, se observan innovaciones y diferencias como la aparición de azadas y cambios morfológicos en algunas puntas de proyectil y el progresivo abandono del uso de láminas. Estas diferencias parecen producirse debido a las nuevas condiciones de vida sedentaria y economía agrícola y pastoril.

Los cambios tecnológicos parecen corresponder con modificaciones en el sistema de asentamiento, inicialmente enfocado en el aprovechamiento de recursos localizados en la orilla del Lago Uru-Uru y posteriormente diversificado hacia áreas interiores y de mayor elevación, incluyendo terrenos de mayor productividad agrícola y pastoril, pero también de mayor facilidad defensiva. Este patrón es comparable con el observado en otras regiones (Aldenderfer 1998; Klink 2005; Santoro y Núñez 1987), pero todavía se requieren más investigaciones incluyendo más sitios excavados, fechados radiocarbónicos y análisis de material arqueológico proveniente de excavaciones arqueológicas para confirmar nuestras observaciones preliminares. Actualmente, nuestro proyecto viene realizando análisis de material arqueológico procedente de excavaciones realizadas en varios sitios de la región, cuyos resultados esperamos puedan complementar la información aquí presentada (Capriles 2011).

\section{Conclusiones}

En este reporte se presenta, por primera vez, evidencia acerca de la transición entre el período Arcaico y el período Formativo en el altiplano boliviano. Los cambios en el patrón de asentamiento observados en la zona de Iroco sugieren que los campamentos del período Arcaico fueron sustituidos por asentamientos de mayor intensidad ocupacional durante el período Formativo. El incremento observado entre sitios ocupados está relacionado con una ampliación y diversificación del paisaje utilizado, evidentemente en correspondencia con nuevas actividades económicas. Más aún, el crecimiento exponencial observado en la cantidad de superficie ocupada sugiere un notable incremento poblacional asociado con la adopción de la agricultura y el pastoralismo de camélidos. La evidencia del material lítico encontrado en estos sitios sugiere una importante continuidad en el aprovechamiento de ciertas materias primas, así como en la manufactura de ciertas herramientas. Aún así, se notan importantes cambios, como ser la diversificación de la tecnología bifacial, incluyendo la manufactura de azadas. El análisis de las excavaciones arqueológicas, actualmente en curso, permitirá tener una mayor claridad cronológica y determinar con mayor precisión cambios específicos asociados con estos períodos. Esperamos en el futuro reportar estos resultados y aportar mayores luces acerca de la región y su cambio a lo largo del tiempo.

Agradecimientos: Agradecemos los comentarios ofrecidos a una versión anterior de este reporte a Mark Aldenderfer, David Browman, Alejandra Domic, Luis Ángel Flores Blanco, Steven Goldstein, Fiona Marshall, Hugo Yacobaccio y un revisor anónimo. La investigación contó con el 
soporte financiero de la Beca BCS\# 0737793 de la National Science Foundation de Estados Unidos. Queremos agradecer el apoyo al proyecto de parte de la Unidad Nacional de Arqueología de Bolivia y de la Empresa Minera Inti Raymi, S.A., particularmente al Lic. Jorge Saavedra, Lic. Marco Luna, Ing. Miguel Reynaga así como a los dirigentes y pobladores de las comunidades de Iroco, Cochiraya y Chuzequeri. De la misma manera, agradecemos a Patricia Álvarez, Alejandro Barrientos, Juan Carlos Segurola, Vidal Colque, Mauricio Rocha, Jorge Ustarez, Armando Vargas, Javier Aguilar, Edwin Choque, Alex Flores e Israel Quispe por su apoyo a lo largo del proyecto.

\section{Referencias Citadas}

Abbott, M.B., M.W. Binford, M. Brenner y K.R. Kelts 1997. A $3500{ }^{14} \mathrm{C}$ yr high-resolution record of water-level changes in Lake Titicaca, Bolivia/Peru. Quaternary Research 47:169-180.

Albarracín-Jordán, J. 1996. Tiwanaku: Arqueología Regional y Dinámica Segmentaria. Plural Editores, La Paz.

- - - 2005. Empresa Minera Inti Raymi, Proyecto Kori Chaca: Estudio de Evaluación de Impacto Arqueológico (EEIAR). Informe presentado a la Unidad Nacional de Arqueología de Bolivia. 2 vols. Fundación Bartolomé de las Casas, La Paz.

Aldenderfer, M.S. 1989. The Archaic Period in the South-Central Andes. Journal of World Prehistory 3:117-158.

- - - 1998. Montane Foragers: Asana and the South-Central Andean Archaic. University of Iowa Press, Iowa City.

Andrefsky, W. Jr. 2005. Lithics: Macroscopic Approaches to Analysis, 2da edición. Cambridge University Press, Cambridge.

- - - 2009. The analysis of stone tool procurement, production, and maintenance. Journal of Archaeological Research 17:65-103.

Ayala, P. y M. Uribe 2003. La cerámica Wankarani y una primera aproximación a su relación con el Período Formativo del Norte Grande de Chile. Textos Antropológicos 14(2):7-29.

Ayala, P., C. Carrasco y M. Uribe 2008. Alfarería y líticos Wankarani: caracterización y vínculos con el Norte Grande de Chile. En Arqueología de las Tierras Altas, Valles Interandinos y Tierras Bajas de Bolivia: Memorias del I Congreso de Arqueología de Bolivia, editado por C. Rivera Casanovas, pp. 99-114. Universidad Mayor de San Andrés, Programa de Investigación Estratégica en Bolivia, La Paz.

Baucom, P.C. y C.A. Rigsby 1999. Climate and lake-level history of the northern Altiplano, Bolivia, as recorded in Holocene sediments of the Rio Desaguadero. Journal of Sedimentary Research 69:597-611.

Bermann, M. y J. Estévez Castillo 1993. Jachakala: a new archaeological complex of the Department of Oruro, Bolivia. Annals of the Carnegie Museum 62(4):311-340.

- - - 1995. Domestic artifact assemblages and ritual activities in the Bolivian Formative. Journal of Field Archaeology 22:389-398.

Browman, D.L. 1981. New light on Andean Tiwanaku. American Scientist 69(4):408-419.

Burger, R.L., K.L. Mohr-Chávez y S.J. Chávez 2000. Through the glass darkly: Prehispanic Obsidian procurement and ex- change in southern Peru and northern Bolivia. Journal of World Prehistory 14:267-362.

Calla, S.A. 2009. Análisis de Materiales Líticos, Proyecto Arqueológico en Iroco. Informe inédito en posesión de los autores, La Paz.

Capriles, J.M. 2008. Proyecto Arqueológico en Iroco, Temporada 2007. Informe de Avance de Investigación presentado a la Unidad Nacional de Arqueología de Bolivia, La Paz.

- - - 2011. The Economic Organization of Early Camelid Pastoralism in the Andean Highlands of Bolivia. Tesis doctoral inédita. Department of Anthropology, Washington University, St. Louis.

Cipolla, L.M. 2005. Preceramic Period settlement patterns in the Huancané-Putina River Valley, Northern Titicaca Basin, Peru. En Advances in Titicaca Basin Archaeology, editado por C. Stanish, A.B. Cohen y M.S. Aldenderfer, vol. 1, pp. 55-63. Cotsen Institute of Archaeology, University of California, Los Angeles.

Cuenca Sempertegui, A., E. Garnica Bahoz, E. López Canelas e I. Marca Cáceres 2005. Más Allá de las Pajas y Espinas: Biodiversidad en el Municipio de Oruro (Comunidades CochirayaIroco-Chuzekery). CEPA, Latinas Editores, Oruro.

De Souza, P. 2004. Cazadores recolectores del Arcaico Temprano y Medio en la cuenca superior del río Loa: Sitios, conjuntos líticos y sistemas de asentamiento. Estudios Atacameños 27:7-43.

Fox, J.R. 2007. Time and Process in an Early Village Settlement System on the Bolivian Southern Altiplano. Tesis doctoral inédita. Department of Anthropology, University of Pittsburgh, Pittsburgh

Giesso, M. 2003. La Industria Lítica Expeditiva en Sitios Urbanos Tiwanaku, Bolivia. Textos Antropológicos 14(2):31-46.

Giesso, M., M.D. Glascock y M.R. Michel López 2003. Exploring relations between Khonko Wankane and Tiwanaku: basalt procurement and production. Ponencia presentada en la 31st Midwest Conference on Andean and Amazonian Archaeology and Ethnography, Chicago.

Hastorf, C.A. 2008. The Formative Period in the Titicaca Basin. En Handbook of South American Archaeology, editado por H. Silverman y W.H. Isbell, pp. 545-561. Springer, New York.

Ibarra Grasso, D.E. 1965. Prehistoria de Bolivia. Editorial Los Amigos del Libro, La Paz.

Janusek, J.W. 2008. Ancient Tiwanaku. Cambridge University Press, Cambridge. 
Klink, C.J. 2005. Archaic Period research in the Río Huenque Valley, Peru. En Advances in Titicaca Basin Archaeology, editado por C. Stanish, A.B. Cohen y M.S. Aldenderfer, vol. 1, pp. 13-24. Cotsen Institute of Archaeology, University of California, Los Angeles.

Klink, C.J. y M.S. Aldenderfer 2005. A projectile point chronology for the South-Central Andean highlands. En Advances in Titicaca Basin Archaeology, editado por C. Stanish, A.B. Cohen y M.S. Aldenderfer, vol. 1, pp. 25-54. Cotsen Institute of Archaeology, University of California, Los Angeles.

Lizárraga-Mehringer, Y. 2004. Viscachani y el Precerámico en Bolivia. Tesis doctoral inédita. Facultad de Filosofía, Universidad de Colonia, Colonia.

McAndrews, T.L. 1998. Early Village-Based Society and LongTerm Cultural Evolution in the South-Central Andean Altiplano. Tesis doctoral inédita. Department of Anthropology, University of Pittsburgh, Pittsburgh.

- - - 2001. Organización y crecimiento de los sistemas de asentamiento temprano basados en aldeas en el altiplano Andino sur central. Textos Antropológicos 13(1-2):135-145.

- - - 2005. Los Sistemas de Asentamientos Wankarani desde una Perspectiva Evolutiva: Estudio de una Sociedad Temprana Basada en la Aldea y su Evolución Cultural en el Sur del Altiplano Central Andino. Traducido por A.M. Boada Rivas. University of Pittsburgh Memoirs in Latin American Archaeology No. 15, Plural Editores, Pittsburgh.

Michel López, M.R. 2008. Patrones de Asentamiento Precolombino del Altiplano Boliviano. Lugares Centrales en la Región de Quillacas, Departamento de Oruro, Bolivia. Tesis doctoral inédita. Uppsala University, Uppsala.

Núñez, L., M. Grosjean e I. Cartajena 2005. Ocupaciones Humanas y Paleoambientes en la Puna de Atacama. Instituto de Investigaciones Arqueológicas y Museo, Universidad Católica del Norte, Taraxacum, San Pedro de Atacama.

Odell, G.H. 2003. Lithic Analysis. Springer, New York.

Olivera, D. 1997. La importancia del recurso Camelidae en la Puna de Atacama entre los 10.000 y 500 años a.p. Estudios Atacameños 14:29-41.

Patterson, T.C. y R.F. Heizer 1965. A Preceramic stone tool collection from Viscachani, Bolivia. Ñawpa Pacha 3:107-115.

Ponce Sanginés, C. 1970. Las Culturas Wankarani y Chiripa y su Relación con Tiwanaku. Academia Nacional de Ciencias de Bolivia, La Paz.

Ponce Sanginés, C. y G. Mogrovejo Terrazas 1970. Acerca de la Procedencia del Material Lítico de los Monumentos de Tiwanaku. Academia Nacional de Ciencias de Bolivia, La Paz.
Rigsby, C.A., J.P. Bradbury, P.A. Baker, S.M. Rollins y M.R. Warren 2005. Late Quaternary palaeolakes, rivers, and wetlands on the Bolivian Altiplano and their palaeoclimatic implications. Journal of Quaternary Science 20(7-8):671-691.

Rose, C.E. 2001. Household and Community Organization of a Formative Period, Bolivian Settlement. Tesis doctoral inédita. Department of Anthropology, University of Pittsburgh, Pittsburgh.

Sackett, J.R. 1989. Statistics, attributes, and the dynamics of burin typology. Archaeological Papers of the American Anthropological Association 1:51-82.

Santoro, C.M. 1989. Antiguos cazadores de la Puna (9.000 a 6.000 a.C.). En Culturas de Chile Prehistoria: Desde sus Orígenes Hasta los Albores de la Conquista, editado por J. Hidalgo, V. Schiappacasse, H. Niemeyer, C. Aldunate e I. Solimano, pp. 57-80. Editorial Andrés Bello, Santiago.

Santoro, C.M. y L. Núñez 1987. Hunters of the Dry Puna and the Salt Puna in Northern Chile. Andean Past 1:57-109.

Stanish, C. 2003. Ancient Titicaca: The Evolution of Complex Society in Southern Peru and Northern Bolivia. University of California Press, Berkeley.

Stanish, C., R.L. Burger, L.M. Cipolla, M.D. Glascock y E. Quelima 2002. Evidence for early long-distance obsidian exchange and watercraft use from the southern Lake Titicaca Basin of Bolivia and Peru. Latin American Antiquity 13:444-454.

Sylvestre, F., M. Servant, S. Servant-Vildary, C. Causse, M. Fournier y J.P. Ybert 1999. Lake-level chronology on the southern Bolivian altiplano $\left(18^{\circ}-23^{\circ} \mathrm{S}\right)$ during late-glacial time and the Early Holocene. Quaternary Research 51:54-66.

Thompson, L.G., M.E. Davis, E. Mosley-Thompson, T.A. Sowers, K.A. Henderson, V.S. Zagorodnov, P.N. Lin, V.N. Mikhalenko, R.K. Campen, J.F. Bolzan, J. Cole-Dai y B. Francou 1998. A 25,000-year tropical climate history from Bolivian ice cores. Science 282:1858-1864.

Vela, R.A. 1964. Catálogo de la Colección Vela (Prehistoria Americana). En Contribución al XXXVI Congreso Internacional de Americanistas. Diputación Provincial de Valencia. Servicio de Investigación Prehistórica, Valencia.

Walter, H. 1966. Beiträge zur Archäologie Boliviens. Die Grabungen des Museums für Völkerkunde Berlin im Jahre 1958. Archäologische Studien in Kordilleren Boliviens II, Verlag von Dietrich Reimer, Berlin.

Yacobaccio, H.D. y M. Morales 2005. Mid-Holocene environment and human occupation of the Puna (Susques, Argentina). Quaternary International 132(1):5-14. 